\title{
Relapsing Polychondritis Following Primary Sclerosing Cholangitis: A Rare Coincidence or an Intrinsic Risk?
}

\author{
Hua Li ${ }^{1}$, Bin Liu ${ }^{1 *}$, Wen Zhang ${ }^{2}$, Yuan Yao ${ }^{1}$, Liqing Wang ${ }^{1}$, Miaomiao Xin ${ }^{1}$ and Lei Zhao ${ }^{1}$ \\ ${ }^{1}$ Department of Rheumatology and Immunology, The Affiliated Hospital of Medical College Qingdao University, China \\ ${ }^{2}$ Department of Rheumatology, Peking Union Medical College Hospital, Chinese Academy of Medical Science, China
}

"Corresponding author: Liu B, Department of Rheumatology and Immunology, The Affiliated Hospital of Medical College Qingdao University, No.16 Jiang Su Lu, Shi Nan District, Qingdao city, Shandong Province, 266003, China, Tel: +86 (532) 8291-1847; E-mail: binliu72314@163.com

Rec date: April 23, 2014, Acc date: May 28, 2014, Pub date: June 02, 2014

Copyright: (c) $2014 \mathrm{Li} \mathrm{H}$, et al. This is an open-access article distributed under the terms of the Creative Commons Attribution License, which permits unrestricted use, distribution, and reproduction in any medium, provided the original author and source are credited.

\begin{abstract}
Primary Sclerosing Cholangitis (PSC) is a rare chronic cholestatic liver disease characterized by inflammation and fibrosis of the bile ducts, resulting in end-stage liver disease. It may coexist with other autoimmune diseases. Relapsing Polychondritis (RP) is an uncommon and severe disease which may lead to destruction of elastic cartilages. A case of RP associated with PSC is presented. Although the occurrence of these diseases together could be coincidental, an association seems immunologically plausible.
\end{abstract}

Keywords: Relapsing polychondritis; Primary sclerosing cholangitis; Biliary; Liver

\section{Introduction}

Primary Sclerosing Cholangitis (PSC) is a chronic cholestatic liver disease characterized by concentric and obliterative fibrosis of the intrahepatic and/or extrahepatic bile ducts and a lymphocytic portal tract inflammation leading to cholestasis, cholangitis, and cirrhosis. Either a history of or co-existent Inflammatory Bowel Disease (IBD) has been found in $75-90 \%$ of patients with PSC [1]. Relapsing Polychondritis (RP) is an uncommon condition of unknown etiology characterized by inflammation of cartilage throughout the body, including auricular, nasal, tracheobronchial and articular cartilage $[2,3]$, and approximately $30 \%$ of the cases are associated with systemic non-organ-specific connective tissue diseases, most often a vasculitis and, with systemic organ-specific autoimmune diseases such as thyroid or IBD [4]. A case of PSC followed by RP was presented here and speculation about the relationship of these diseases was offered. To our knowledge, this is a unique case as no other cases with a similar pattern have been reported.

\section{Case Report}

A previously healthy 75 -year-old man presented with pruritus and weight loss, without abdominal pain and jaundice about three years. The patient was admitted to the hospital for operation due to suspected diagnosis Cholangio carcinoma (CCA). During the hospitalization, he suffered from fever, nasal pain, swelling and redness of the external ears. Meanwhile his eyes were pain and photophobia. On clinical examination, he was dry, flushed, febrile, and tachycardia. Scratch marks found on skin. Bilateral helix was swelling, erythema, and tenderness. Eyes were red and nose was tenderness. No other clinical signs of chronic liver disease. Chest was clear, and heart sounds were normal. Abdominal examination revealed soft abdomen with right upper quadrant tenderness and normal bowel sounds. No evidence of ascites, splenomegaly or pedal edema. Ophthalmologic examination showed conjunctivitis. Routine laboratory tests revealed normal blood count, renal functions, and electrolytes. Urinalysis showed positive urobilinogen. ESR was $125 \mathrm{~mm} / \mathrm{hr}$. Liver function tests showed predominantly cholestatic picture with elevated Alkaline Phospatase (ALP) at $176 \mathrm{IU} / \mathrm{L}, \gamma$-Glutamyl Transpeptidase $(\gamma$-GT) at $137 \mathrm{IU} / \mathrm{L}$ and bilirubin at $32 \mu \mathrm{mol} / \mathrm{L}$, but the synthetic liver function increased slightly, such as aspartate aminotransferase (AST) at106 $\mathrm{IU} / \mathrm{L}$, alanine aminotransferase (ALT) at $76 \mathrm{IU} / \mathrm{L}$ and albumin was 24g/L. PT, APTT were normal. Rheumatoid factor (RF), antiextractable nuclear antigen antibodies (ENA), Antineutrophil Cytoplasmic Antibody (ANCA), Anti mitochondrial Antibodies (AMA), anti-smooth muscles antibodies (SMA), anti-liver cell membrane antibodies (LMA), anti-soluble liver cell antibodies (SLA), anti-liver-kidney microsomes antibodies (LKM) were all negative except weakly positive ANA (1:100 granular pattern) and increased IgG at $16.1 \mathrm{~g} / \mathrm{L}$ as well as $\operatorname{IgA}$ at $4.19 \mathrm{~g} / \mathrm{L}$. Serum levels of tumor markers, including CEA, AFP, CA125, CA242, CA19-9, and CA153 were all within the normal ranges. Tc-99m MDP bone scintigraphy showed diffusely increased accumulation of radioactivity in nose, costocartilages, sternoclavicular and ankle joints (Figure1).

An ultrasound scan showed the intrahepatic bile ducts dilatation. Computed Tomography (CT) showed multiple dilatations of the intrahepatic bile ducts in the liver, without cirrhosis. Magnetic Resonance Cholangio Pancreatography (MRCP) showed multiple dilatations and beads like intrahepatic biliary ducts (Figure $2 \mathrm{~A}$ and $2 \mathrm{~B})$. The patient was diagnosed as PSC and RP based on clinical manifestations, laboratory as well as MRCP. His symptoms were markedly improved after treated with prednisone $0.5 \mathrm{~kg} / \mathrm{mg} / \mathrm{d}$ and methotrexate $10 \mathrm{mg} / \mathrm{w}$. After 3 months of treatment, his symptom was completely relieved. Repeated liver function test was normal except slightly elevated $\gamma$-GT at $106 \mathrm{IU} / \mathrm{L}$. After following three years, repeated CT and MRCP showed no remarkably changes (Figures 2C and 2D). 
Citation: Li H, Liu B, Zhang W, Yao Y, Wang L, et al. (2014) Relapsing Polychondritis Following Primary Sclerosing Cholangitis: A Rare Coincidence or an Intrinsic Risk?. J Diabetes Metab 5: 380. doi:10.4172/2155-6156.1000380

Page 2 of 3

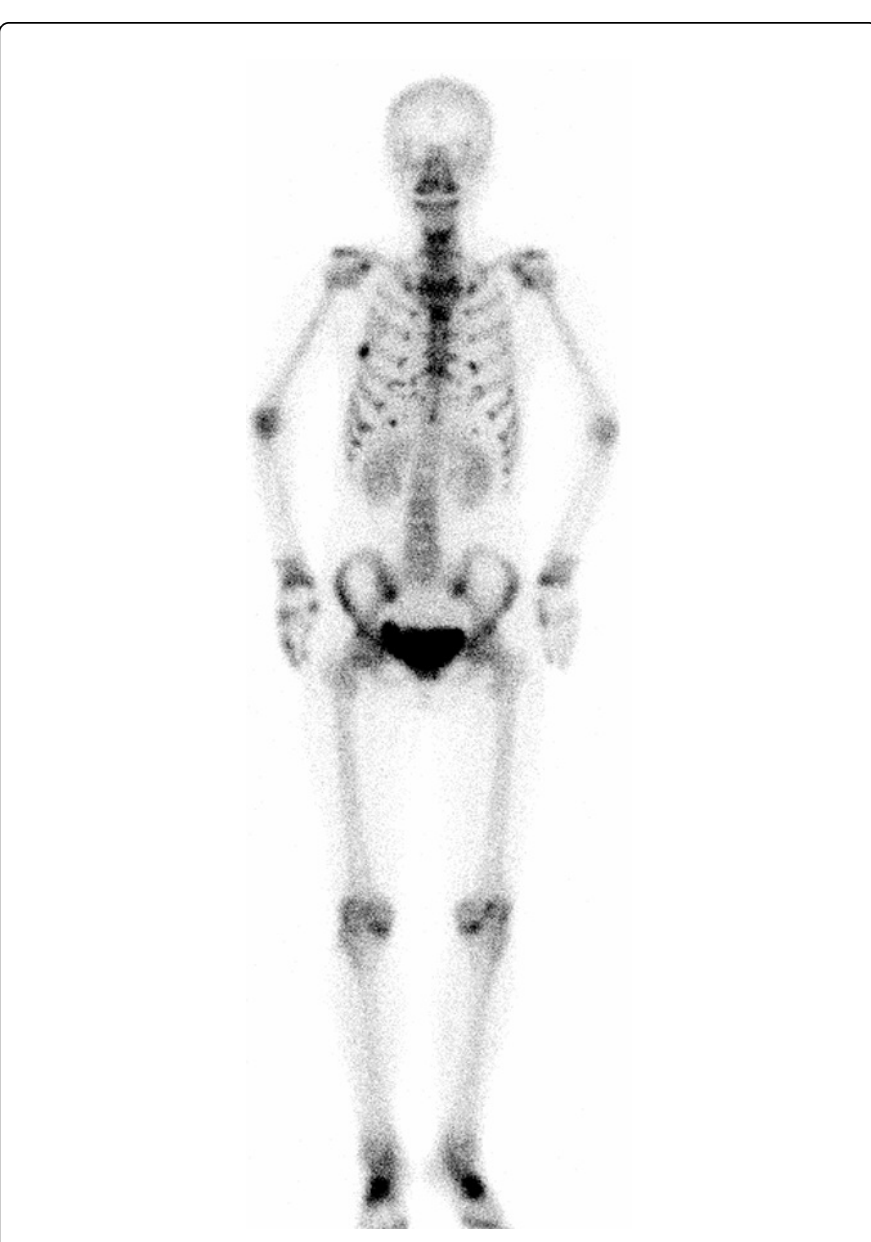

Figure 1: Tc-99m MDP bone scintigraphy demonstrate intense uptake nose, bilateral chest wall and ankle joints.

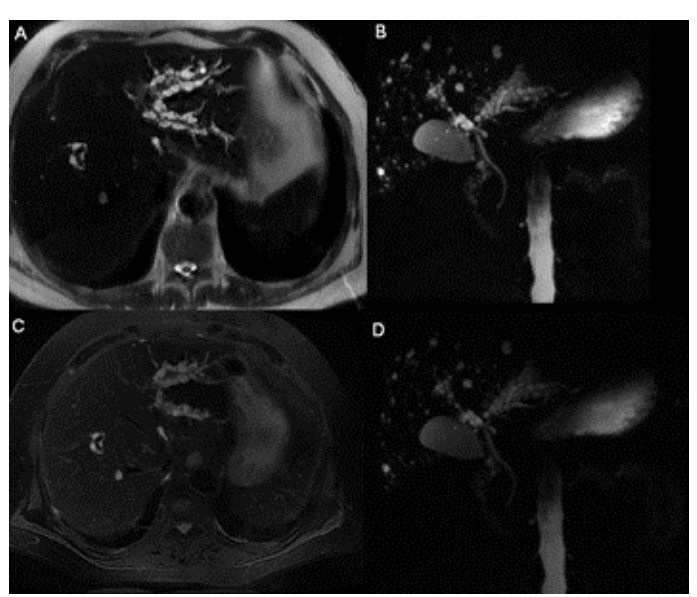

Figure 2: A MRCP (T2-weighted image) reveals typical beading appearance of dilated intrahepatic bile ducts characteristic of PSC. B. MRCP (3D image). C and D After following 3 year repeated MRCP showed no remarkably changes.

\section{Discussion}

The diagnosis of PSC is based on a combination of cholestatic parameters and typical cholangiographic findings, except secondary sclerosing [5]. To data, MRCP has emerged as the standard procedure for detecting PSC. Only the MRCP findings are normal, liver biopsy is required to confirm the diagnosis [6]. The prognosis of PSC is usually poor and liver failure occurs 10-12 years after the onset of PSC. In addition to cirrhosis, approximately $10-15 \%$ of PSC patients will develop CCA, which is the most feared complication often leading to death within a few months [7]. Sudden progressive jaundice, elevated concentration of CA 19-9, weight loss and abdominal pain are frequently associated with the development of CCA in PSC [8]. MRCP is considered the modality of choice in the diagnosis of CCA because of its high contrast resolution, multiplanar capability, and its ability to determine the parenchymal, biliary, and vascular extension [9]. In a clinical setting, IgG4-related sclerosing cholangitis resemble PSC, making diagnosis difficult. The diffusely enlarged pancreas imaging findings and elevated serum IgG4 will be helpful for differential diagnosis [10]. This case illustrated the classic clinical features and a cholestatic pattern on serum liver biochemistry. And MRCP showed intrahepatic ductal dilatation with tortuous and diffuse dilatation most consistent with PSC. Then symptoms and hepatobiliary enzymes got markedly improved with the therapy. After 3 years follow-up, the benign course corroborated a diagnosis of PSC.

PSC might be an immune-mediated process and is indeed associated with other autoimmune diseases [11]. The vast majority of PSC cases occur in the setting of IBD and its association with IBD is well recognized, especially primarily Ulcerative Colitis (UC). Although PSC occurs in approximately $5 \%$ of all patients with UC, up to $75 \%$ of patients with PSC will have associated UC [12]. PSC is also associated with autoimmune disease outside the gastrointestinal tract such as Rheumatoid Arthritis (RA), Sjögren's Syndrome (SS), Systemic Sclerosis (SSc), and Systemic Lupus Erythematosus (SLE) [13]. However, to our knowledge there are no reports of RP in patients with PSC.

RP mainly affects cartilaginous as well as proteoglycan-rich tissues. Up to $70 \%$ of patients with RP have disease limited to inflammatory changes of the cartilages of the head and neck. A small percentage of patients will develop multi systemic involvement, although hepatic involvement in RP is rare. Approximately $30 \%$ cases of RP have coexistent other autoimmune disorders like systemic vasculitis, RA, SLE, SS, and IBD. The diagnosis of RP usually depended on a constellation of clinical features and symptom-driven diagnostic testing. McAdam et al. [14] suggested 6 clinical diagnostic criteria. The presence of 3 out 6 criteria confirmed the diagnosis but histology was not required. The modified criteria were proposed by Damiani and Levine, which required either on three McAdam criteria or at least one McAdam criterion with histologic confirmation, or chondritis at least in two distinct locations with therapeutic response [15]. According to Michet et al. [16], RP diagnosis relied on a proven inflammation in 2 out of 3 auricular, nasal or laryngotracheal cartilages; alternatively, a proven inflammation in one of these cartilages and 2 other signs amongocular inflammation, hearing loss, vestibular dysfunction, or seronegative arthritis were required [2,14-16]. With development of nuclear medicine, positron emission tomography (PET)/CT and Tc-99m MDP bone scintigraphy as well as Ga-67 citrate scintigraphy has emerged as a helpful tool in early diagnosis, determining the biopsy site, assessing inflammatory activity and monitoring a treatment response $[17,18]$. Our patient should be considered have RP 
due to chondritis in two distinct locations, and a rapid response to the administration of prednisone as well as inflammatory cartilage in Tc-99m MDP bone scintigraphy.

Notwithstanding the cause of both RP and PSC is largely unknown, there is evidence of immune system abnormalities and of genetic influences in these disorders. Six different HLA molecules have so far been associated with PSC [19]. Among them, HLA-DR4 might be a marker for rapid disease progression in PSC, and the DR3, DQ2 heterozygous genotype is also associated with a more rapid progression of PSC, whereas HLA-DQ6 is associated with a retarded disease progression $[13,20]$. In RP, Genome-wide association analysis reveals susceptibility to RP is associated with HLA-DR4 [21]. Transgenic strains expressing the DQ6alpha8beta transgene develop spontaneous polyarthritis, auricular chondritis and nasal chondritis at the mouse equivalent of human middle age [22]. Immunogenetic findings suggest similar HLA molecules might play a role in the pathogenesis of both diseases. Data from animal model and human research show that RP might be a Th1-mediated disease as serum levels of Interferon (IFN)- $\gamma$, interleukin (IL)-12, and IL-2 coincident changes in disease activity, but the levels of Th2 cytokines do not [23]. While the lymphocytic infiltrate of PSC also show to be primarily Th1 phenotype, and Th1 cytokines are the predominant type in PSC [24]. Like PSC, RP also always coexisting with UC [4]. Moreover, ANCA could play a role in both RP and PSC pathogenesis. They seem present in $24 \%$ of RP patients and correlated with disease activity [23]. Similarly, they are used as a prognosis marker in PSC $[23,24]$. Therefore, RP associated with PSC might not only be a rare coincidence but also seems intrinsic relationship.

In conclusion, we reported a patient with the extremely rare association of PSC and RP. When patient with RP presents liver function abnormalities, PSC should not be ignored.

\section{Funding Source Statement}

The investigation was supported by grants from Natural Sciences Foundation of China (No.81241094) and Science and Technology program of basic research projects of Qingdao city (NO.13-1-4-138jch).

\section{References}

1. Lutz H, Trautwein C, Tischendorf JW (2013) Primary sclerosing cholangitis: diagnosis and treatment. DtschArzteblInt 110: 867-874.

2. Cantarini L, Vitale A, Brizi MG, Caso F, Frediani B, et al. (2014) Diagnosis and classification of relapsing polychondritis. J Autoimmun 48-49: 53-9.

3. Giroux L, Paquin F, Guérard-Desjardins MJ, Lefaivre A (1983) Relapsing polychondritis: an autoimmune disease. Semin Arthritis Rheum 13: 182-187.

4. Almadi MA, Alenizi AT, Menard HA, Hilzenrat N (2010) Relapsing polychondritis and ulcerative colitis. Saudi J Gastroenterol 16: 49.

5. Karlsen TH, Vesterhus M, Boberg KM (2014) Review article: controversies in the management of primary biliary cirrhosis and primary sclerosing cholangitis. Aliment PharmacolTher 39: 282-301.
6. Dave M, Elmunzer BJ, Dwamena BA, Higgins PD (2010) Primary sclerosing cholangitis: meta-analysis of diagnostic performance of MR cholangiopancreatography. Radiology 256: 387-396.

7. Tanaka A, Takamori Y, Toda G, Ohnishi S, Takikawa H (2008) Outcome and prognostic factors of 391 Japanese patients with primary sclerosing cholangitis. Liver Int 28: 983-989.

8. Levy C, Lymp J, Angulo P, Gores GJ, Larusso N, et al. (2005) The value of serum CA 19-9 in predicting cholangiocarcinomas in patients with primary sclerosing cholangitis. Dig Dis Sci 50: 1734-1740.

9. Malhi H, Gores GJ (2006) Cholangiocarcinoma: modern advances in understanding a deadly old disease. J Hepatol 45: 856-867.

10. Okazaki K, Uchida K, Koyabu M, Miyoshi H, Ikeura T, et al. (2014) "IgG4 Cholangiopathy - current concept, diagnosis and pathogenesis " J Hepatol .

11. O'Mahony CA, Vierling JM (2006) Etiopathogenesis of primary sclerosing cholangitis. Semin Liver Dis 26: 3-21.

12. Loftus EV Jr, Harewood GC, Loftus CG, Tremaine WJ, Harmsen WS, et al. (2005) PSC-IBD: a unique form of inflammatory bowel disease associated with primary sclerosing cholangitis. Gut 54: 91-96.

13. Gow PJ, Fleming KA, Chapman RW (2001) Primary sclerosing cholangitis associated with rheumatoid arthritis and HLA DR4: is the association a marker of patients with progressive liver disease? J Hepatol 34: 631-635

14. McAdam LP, O'Hanlan MA, Bluestone R, Pearson CM (1976) Relapsing polychondritis: prospective study of 23 patients and a review of the literature. Medicine (Baltimore) 55: 193-215.

15. Damiani JM, Levine HL (1979) Relapsing polychondritis--report of ten cases. Laryngoscope 89: 929-946.

16. Michet CJ Jr, McKenna CH, Luthra HS, O'Fallon WM (1986) Relapsing polychondritis. Survival and predictive role of early disease manifestations. Ann Intern Med 104: 74-78.

17. Chang MC, Tsai SC, Lin WY (2008) Value of gallium-67 scanning in monitoring therapeutic effectiveness in a patient with relapsing polychondritis. Kaohsiung J Med Sci 24: 328-333.

18. Yamashita H, Takahashi H, Kubota K, Ueda Y, Ozaki T, et al. (2014) Utility of fluorodeoxyglucose positron emission tomography/computed tomography for early diagnosis and evaluation of disease activity of relapsing polychondritis: a case series and literature review. Rheumatology (Oxford).

19. Donaldson PT (2004) Genetics of liver disease: immunogenetics and disease pathogenesis. Gut 53: 599-608.

20. Boberg KM, Spurkland A, Rocca G, Egeland T, Saarinen S, et al. (2001) The HLA-DR3,DQ2 heterozygous genotype is associated with an accelerated progression of primary sclerosing cholangitis. Scand J Gastroenterol 36: 886-890.

21. Zeuner M, Straub RH, Rauh G, Albert ED, Schölmerich J, et al. (1997) Relapsing polychondritis: clinical and immunogenetic analysis of 62 patients. J Rheumatol 24: 96-101.

22. Lamoureux JL, Buckner JH, David CS, Bradley DS (2006) Mice expressing HLA-DQ6alpha8beta transgenes develop polychondritis spontaneously. Arthritis Res Ther 8: R134.

23. Arnaud L, Mathian A, Haroche J, Gorochov G, Amoura Z (2014) Pathogenesis of relapsing polychondritis: a 2013 update. Autoimmun Rev 13: 90-95.

24. Aron JH, Bowlus CL (2009) The immunobiology of primary sclerosing cholangitis. SeminImmunopathol 31: 383-397. 